\section{Facharztprüfung zur Erlangung des Facharzttitels FMH für Rheumatologie}

Aufgrund des Weiterbildungsprogrammes, welches am 1. Januar 1996 in Kraft gesetzt wurde, ist die Teilnahme an der Facharztprüfung für diejenigen Kandidatinnen und Kandidaten Voraussetzung für die Erlangung des Facharzttitels FMH für Rheumatologie, welche ihre Weiterbildung in Rheumatologie bis am 31. Dezember 1996 nicht abgeschlossen hatten. Es empfiehlt sich, die Facharztprüfung frühestens im letzten Jahr der reglementarischen Weiterbildung abzulegen (Art. 22 WBO).
Schriftliche Prüfung

Ort: Hotel Bern, Bern

Datum: Mittwoch, 17. Januar 2001

Prüfungsgebühr: Die Fachgesellschaft erhebt für die schriftliche Prüfung eine Prüfungsgebühr von Fr. 300.-.

Schriftliche Anmeldung:

Péter Tamás, Sekretariat SGR

c/o Schweiz. Rheumaliga

Renggerstrasse 71, Postfach, 8038 Zürich

Tel. 0148740 00/07, Fax 014874019

E-mail:pt@srl.ch

Anmeldefrist: 30. November 2000

\section{Facharztprüfung zur Erlangung des Untertitels Handchirurgie zu den Facharzttiteln FMH für Chirurgie, Kinderchirurgie, orthopädische Chirurgie sowie Plastische und Wiederherstellungschirurgie}

Aufgrund des Weiterbildungsprogrammes, welches am 1. Juli 1996 in Kraft gesetzt wurde, ist die Teilnahme an der Facharztprüfung für diejenigen Kandidatinnen und Kandidaten Voraussetzung für die Erlangung des Untertitels Handchirurgie zum Facharzttitel FMH für Plastische und Wiederherstel- lungschirurgie. Es empfiehlt sich, die Facharztprüfung frühestens im letzten Jahr der reglementarischen Weiterbildung abzulegen (Art. 22 WBO).

Ort und Zeitpunkt: Universitätsspital Zürich, Klinik für Wiederherstellungschirurgie, Rämistrasse 100 , 8091 Zürich

1. Termin: 24. November 2000

2. Termin: 6. April 2001

Prüfungsgebühr: Die Fachgesellschaft erhebt einen Unkostenbeitrag von CHF 500.-.

Anmeldung:

Dr. W. Künzi, Leitender Arzt

Universitätsspital Zürich

Klinik für Wiederherstellungschirurgie

Rämistrasse 100, 8091 Zürich

Tel. 0125527 07, Fax 012558948

E-mail doris.schreiber@chi.usz.ch

Anmeldefrist: 15. Oktober 2000 


\section{Examen de spécialiste en vue de l'obtention du titre de spécialiste FMH en rhumatologie}

Conformément au programme de formation postgraduée qui est entré en vigueur le $1^{\mathrm{er}}$ janvier 1996, la participation à l'examen de spécialiste est obligatoire pour les candidats à l'obtention du titre FMH en rhumatologie qui n'avaient pas terminé leur formation postgraduée en rhumatologie le 31 décembre 1996. Il est recommandé de passer l'examen de spécialiste au plus tôt durant la dernière année de la formation postgraduée réglementaire (art. 22 RFP).
Examen écrit

Lieu: Hôtel Bern, Berne

Date: Mercredi, 17 janvier 2001

Taxe d'examen: La société de discipline médicale prélève une participation aux frais de Fr. 300.- pour l'examen écrit.

Inscriptions (par écrit):

Péter Tamás, Secrétariat SSR

c/o Schweiz. Rheumaliga

Renggerstrasse 71, Case postale, 8038 Zurich

tél. 0148740 00/07, fax 014874019

e-mail:pt@srl.ch

Délais d'inscription: le 30 novembre 2000
Examen de spécialiste en vue de l'obtention du sous-titre en chirurgie de la main de spécialiste FMH en chirurgie, chirurgie pédiatrique, chirurgie orthopédique et chirurgie plastique et reconstructive

Conformément au programme de formation postgraduée entré en vigueur le $1^{\text {er }}$ juillet 1996, la participation à l'examen de spécialiste est une condition requise pour les candidats à l'obtention du sous-titre en chirurgie de la main de spécialiste FMH en chirurgie plastique et reconstructive. Il leur est recommandé de passer l'examen de spécialiste au plus tôt durant la dernière année de leur formation postgraduée réglementaire (art. 22 RFP).

Lieu et date: L'Hôpital universitaire, Clinique de Chirurgie reconstructive, Rämistrasse 100, 8091 Zurich

$1^{\text {re }}$ date: le 24 novembre 2000

$2^{\mathrm{e}}$ date: le 6 avril 2001

Taxe d'examen: La société de discipline médicale prélève une participation aux frais de CHF 500.-.

\section{Inscription:}

auprès du Dr W. Künzi

L'Hôpital universitaire

Clinique de Chirurgie reconstructive

Rämistrasse 100, 8091 Zurich

tél. 0125527 07, fax 012558948

e-mail doris.schreiber@chi.usz.ch

Délai d'inscription: le 15 octobre 2000 